\title{
DWT with Enhanced ANN Technique for Detecting and Classifying the Faults of Synchronous Generator
}

\author{
Boorgula Vidyasagar $^{1^{*}} \quad$ Sri Tulasi Ram Sankara ${ }^{2}$ \\ ${ }^{I}$ Trinity College of Engineering and Technology, Karimnagar, Telangana, India \\ ${ }^{2}$ Jawaharlal Nehru Technological University, College of Engineering Kukatpally, Hyderabad, India \\ Corresponding author's Email: bvidyasagar1072@gmail.com
}

\begin{abstract}
In the paper, a hybrid technique based fault detector algorithm for synchronous generator is proposed. The hybrid technique is the combination of Artificial Neural Network (ANN) and Gravitational Search Algorithm (GSA). The GSA is used to train the ANN and improve the performance of ANN. Initially, the synchronous generator is analyzed in the normal condition. After that, the fault is created in the synchronous generator and the system behaviors are monitored and signals are measured which can be seen as distorted waveforms. These distorted waveforms are composed of different frequency components and which are needed to be represented in timefrequency domain for fault analysis. For this representation of signal, discrete wavelet transform (DWT) is presented. It extracts the features and forms the datasets which are forwarded to ANN classifier for classifying the type of fault occurred in the stator winding of the synchronous generator. In order to evaluate the effectiveness of the proposed method, the internal faults are analyzed. The proposed technique is implemented in MATLAB/simulink platform and this is validated using statistical measures such as accuracy, sensitivity \& specificity. The proposed method is compared with the existing techniques DWT-ANN with GA and DWT-ANN methods.
\end{abstract}

Keywords: synchronous generator; Internal fault; ANN; DWT; GSA and Stator winding current.

\section{Introduction}

The expansion of trade is openly associated to its energy utilization and electricity. It is essential to contain a consistent power system, both in the production and moving of electric energy to preserve and augment the expansion phase [1]. Generators are in the middle of the most significant components of a power system and their rapid and safe guard is critical [2]. In order to achieve variable speed process and to satisfy the network needs with a high presentation, in generator drives with fullscale power converters, blocked loop manage systems are compulsory [3]. The synchronous generator is indicated as a broad diversity of irregular functions and mistakes [4]. Besides, medium and large sized synchronous generators are stranded by high impedance, whose value is usually put so as to bind the neutral current [5]. State observes of the synchronous generators to tackle this mission, because the probable rotor mistakes have been selected as an idea in this absorb. To complete this objective, safety should exactly identify mistakes [6].

Although, it holds some probable protection problems such as irretrievable demagnetization in rotor permanent magnets (PMs) according to the temperature increase and demagnetizing current, unbalanced injure to PM, inequitable short circuit in stator winding, and open circuit [7]. Moreover, to identify with predictable defense strategy such as over current and discrepancy defense, this type of error is complicated because the current sensors, typically situated at the external stator winding cannot compute an interior short-circuit loop current [8]. To dynamic rectifiers, a relative study of error analytic method is applied, where a few techniques were projected that depends on a variety of limitations [9]. A few of the On-line methods that have been classifying framework winding position and stage mistakes with region current observing, dimension of third harmonic voltages and artificial neural networks.

In general, the current error recognition and analysis method usually depends on intellectual 
systems using traditional methods in time or frequency field, and arithmetical analysis. Fuzzy, ANN, GA techniques as well as intellectual hybrid systems such as fuzzy-GA, Nero-fuzzy, Nero-GA systems can be considered as an intelligent error analysis systems. For online error analysis, the examination of machine signals, combined with the Artificial Intelligence (AI), has been one of the regularly used approaches. These signals could be thermal, magnetic field, flux density, motor currents, voltages or mechanical torque, vibration [10]. In significantly recovering the accuracy in error recognition technique, AI-based methods have the possible benefit above predictable techniques [11]. Therefore, an efficient technique is needed to analyze the synchronous generator faults. In the paper, a hybrid technique is utilized to analyze synchronous generator faults. The hybrid technique is developed for detecting and classifying the synchronous generator faults. The proposed methods are made clear in section 3. The most recent research works are disputed in section 2 . The results and discussion of the proposed strategy is described in section 4 . The section 5 finishes the document.

\section{Recent Research Works: A Brief Review}

Numerous related works are already existed in literatures which are based on fault diagnosis in electrical distribution system. Some of them are reviewed here.

R. Gopinath et al [12] have proposed a condition based maintenance (CBM) that needs uninterrupted observing of mechanical/electrical signals and a variety of functioning setting of the machine which deals an upholding choice.

X. L. Zhang et al [13] have projected the expansion of a consistent and competent intellectual error analysis method which was an incomplete effort. Support vector machine (SVM) is a generally used machine learning system in intellectual error analysis.

P. M. Vahdati et al [14] have projected a novel voltage-controlled over current defense system as a support defense technique for the discrepancy defense technique. This system was considered to activate the setting in which discrepancy defense lose to do so and was executed by fuzzy controllers. The fuzzy controllers put the plug location of the above current transmit.

G. Niu et al [15] have proposed a vigorous error finding and analytical system. For scheme error representation and a data-driven method for online irregularity observing, it is developed for a multi energy field system that incorporates a model-based approach.

A. Ali et al [16] have proposed a model-based error analysis system. It is developed for finding of errors in power systems. In condition break type, the error representation in power generating units (PGUs) and communication and distribution networks (T\&DN) have been copied. To recognize these errors, an observer-based fault detection filter (FDF) has been utilized.

In the power system network, the protection of synchronous generator from strange conditions is important as it is the most costly hardware. The faults in the synchronous generator are named as inward blames and outside issues [1]. For just 95\% of the stator twisting, the strategies portrayed can distinguish ground issues, as there is insufficient voltage to drive the current operation of transfer. In the paper, to overcome these limitations, a DWT based hybrid technique is proposed.

\section{Fault Detection and Classification of Synchronous Generator using Hybrid Method}

In the section, the demonstrating of synchronous generator is described. It is done by considering a simple arrangement of the power system network with synchronous generator and a source. The synchronous generator is sustained to load through a step down transformer and a transmission line. At position $\mathrm{A}$ and $\mathrm{B}$ individually, the inside shortcomings and outer flaws are made with a specific end goal to reconstruct the inward blames.

The stator adding so as to wind shortcomings are made two arrangement voltage sources with converse polarities to each other in the broken stage at the outer terminals. The extent of these voltage sources is equivalent to the electro intention power of the sound bit of the windings. The sub-transient, transient and synchronous reactance of synchronous generator is lessened by an estimation of $\mathrm{x}$ which is equivalent to the sub transient reactance of the solid part of the windings. The inside issues are recreated for single line to ground shortcoming (L-G), line to line deficiency (L-L) and line to line to line flaw (LL-L). With a specific end goal to distinguish the issues in synchronous generator hybrid strategy has been considered.

\subsection{DWT based Hybrid Technique for Fault Detection and Classification}




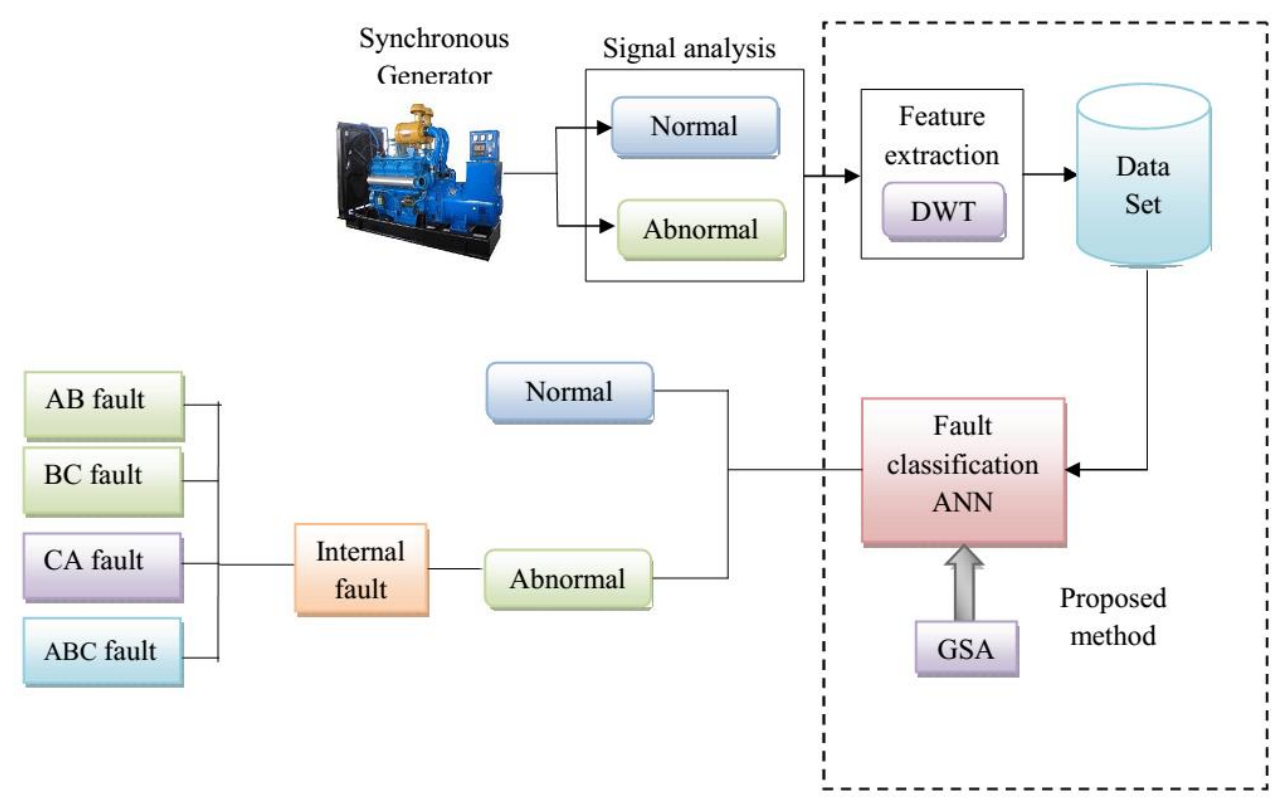

Figure.1 Illustration of Structure in Proposed method with synchronous generator

Here, using the proposed hybrid method, the synchronous generator is analyzed. The hybrid method is the grouping of the Artificial Neural Network (ANN) and Gravitational Search Algorithm (GSA). In the beginning, in normal condition, the performance of synchronous generator is analyzed. Later, the fault is created in the synchronous generator and classified the faults whether is it internal fault or external. Initially, to extract the features of the faulty or normal signal, the Discrete Wavelet Transform (DWT) is applied. Then using enhanced ANN method the faults are classified. Here, to improve the performance of ANN according to the training process of ANN, GSA algorithm is utilized. The detailed description of the proposed method is explained in the following section.

\subsubsection{Feature extraction using Discrete Wavelet Transform}

The discrete wavelet transform (DWT), signifies a linear alteration which functions on a data vector whose length is an integer power of two, transforming it into a numerically diverse vector of identical length. For successive evaluation of each module with resolution corresponding to its scale, it is an amazing device that classifies data into various frequency segments $[17,18]$. In this paper, for creating the datasets for classifying the faults according to their frequencies, DWT is used. Here, the original and fault transient current signals of synchronous generators are decomposed into two components, high and low-frequency components.
The high frequency component of the signal is signified as low-scale decomposition which is denoted as detailed coefficients and the lowfrequency component of the signals is identified as high-scaled decomposition which is called as approximate coefficients. To reduce the difficulty involved due to current magnitudes, then the signals should be normalized. In the course of stable state functioning of synchronous generator, stator winding currents are normalized to their assessed peak value. To evaluate the synchronous generator faults of diverse assessed values, the plus factor of normalization is characterized by its competence.

$$
X_{C_{p}}^{d w t}\left[X_{C_{p}}(t)\right]=\int X_{C_{p}}(t) \lambda(m, n) d t
$$

From the above equation (1) represents the stator winding current signals of synchronous generator using DWT.

Where, $\mathrm{X}_{\mathrm{cp}}(\mathrm{t})$ is denoted as normalized current signal at three phases then, $\lambda(m, n)$ is specified as the basic analyzing function. The mother wavelet is defined as follows:

$$
\lambda(m, n)=\left(\frac{1}{\sqrt{a_{0}^{m}}} \lambda\left(\frac{t-n a_{0}^{m} b_{0}}{a_{0}^{m}}\right)\right)
$$

Where, $\mathrm{m}$ and $\mathrm{n}$ are integers and $a=a_{0}^{m}$ and $b=n a_{0}^{m} b_{0}$ are denoted as the real numbers which represent the scaling (dilation) and translation (shift) parameters. In DWT, after sampling, the normalized stator winding current signals passes through two complementary filters, namely low-pass and high- 
pass filters and emerges as two signals, called approximation coefficients, $\mathrm{X}_{\mathrm{p}}^{\mathrm{a}, \mathrm{DWT}}$ and detail coefficients, $X_{\mathrm{p}}^{\mathrm{d} \text {,DWT }}$.

With the use of DWT, two types of data are collected in normal and faulty case such as, low and high frequency signal data. If there is any fault condition present in the synchronous generator, it forwards the current wavelet coefficients for classification of fault type. The output of the DWT algorithm described as the following equations, which contains the low and high frequency datasets of current.

$$
D S_{d w t}=\left[\begin{array}{c}
L F^{1} \\
L F^{2} \\
\cdot \\
\cdot \\
\cdot \\
L F^{n}
\end{array}\right]^{c, v}\left[\begin{array}{c}
H F^{1} \\
H F^{2} \\
\cdot \\
\cdot \\
\cdot \\
H F^{n}
\end{array}\right]^{c, v}
$$

Where, $L F_{n}^{c, v}$ and $H F_{n}^{c, v}$ are specified as the low $\&$ high frequency current signals. These datasets are given to the input of the ANN. The detailed procedure of the ANN is described in this following section.

\subsection{Hybrid Technique for Fault Classification of Synchronous Generator}

The hybrid technique is utilized for classifying the internal failures of the synchronous generators, in the paper. The hybrid technique is the combination of the ANN and GSA. The traditional ANN is training done by using back propagation algorithm. But in the paper, GSA algorithm is used to train the ANN. The performance of the ANN is improved, by utilizing the GSA. The proposed hybrid method is described as below,

\subsubsection{ANN with Aid of GSA}

ANNs are a well approach for developing the mathematical structures with the ability to learn. It has the notable capability to derive meaning from complicated or indefinite data. This can be used help to extract patterns and detect trends that are excessively complex to be noticed by other techniques [19]. BP technique is based on gradient descent method. The concept is to have an error function and use hill climbing or descent to find the weights. This would optimize the task at hand. Though studies have established that back propagation is prone to the following problems - it may get stuck at a local optimum and it may take a very long time to converge.

International Journal of Intelligent Engineering and Systems, Vol.9, No.4, 2016

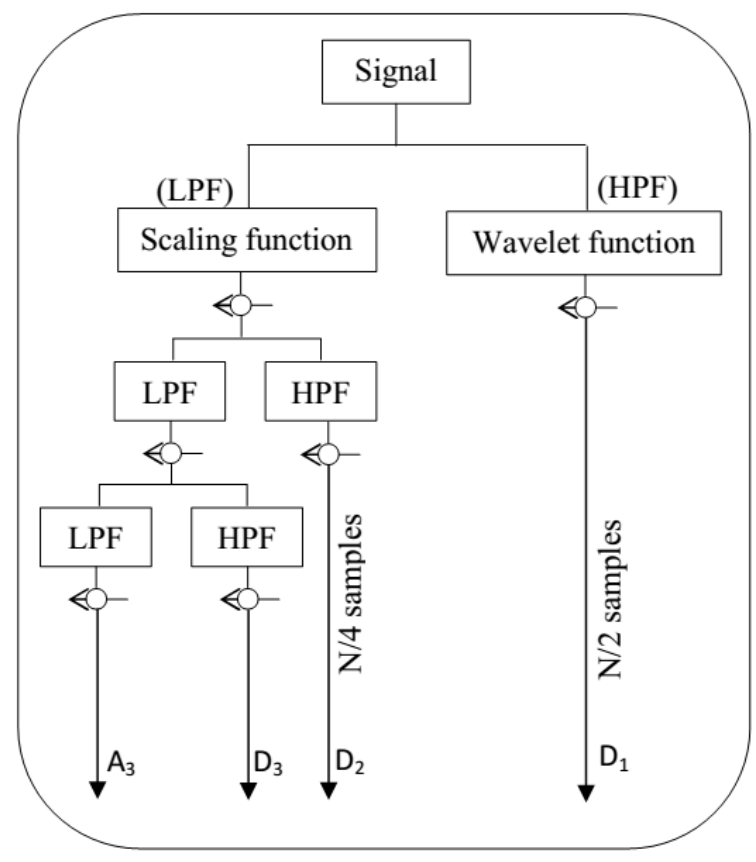

Figure.2 Block diagram of DWT

This led researchers to attack the ANN training problem with other methods. Another technique for ANN weight optimization is by using Genetic algorithms (GA). GA encodes the ANN weights as possible solutions for the problem in the chromosomes of simulated biological organisms. In each generation the organisms with best chromosomes are chosen for reproduction. To regulate the outcome of the final result, the probability of crossover and mutation can be adjusted [20]. This continues for a predefined number of generations or until the problem is sufficiently optimized.

Compared to BP, GA has parallel search strategy and global optimization characteristics which helps the ANN to have a higher prediction accuracy and faster convergence. However the genetic operators like crossover and mutation are inherently complex and hence make the computational cost to increase exponentially. The convergence speed of GA, though better than BP, is still slow and even stagnates as it approaches the optimum. In the paper, the ANN is trained by using GSA, which is used for classifying the synchronous generator faults. Here, the normal and abnormal data are initially evaluated to determine its integrity. The input of the ANN is the stator winding current. The ANN is designed and trained to produce the output vector. Once trained, through its hidden layers, the ANN develops a correlation among all inputs.

Normally the ANN have three layers that is given by, 
(i) Input layer,

(ii) Hidden layer,

(iii) Output layer.

The proposed ANN has the internal fault current $(C)$ as an input and $h$ number of hidden layers. Here, ANN is utilized to classify the fault type whether the synchronous generator is normal or abnormal.

The proposed ANN structure is trained by GSA technique and observes the changes to prediction accuracy. The training algorithm steps are described as below. Here, the inputs are considered as the $\left(X_{1}, X_{2}, X_{31} \ldots \ldots X_{n}\right)$ values and the weight of the network is assigned for input layer to hidden layer and hidden layer to output layer. From the input layer to hidden layer weights are denoted as $\left(\mathrm{w}_{11}, \mathrm{w}_{12}, \ldots \mathrm{w}_{1 \mathrm{n}}\right), \quad\left(\mathrm{w}_{21}, \mathrm{w}_{22}, \ldots . . \mathrm{w}_{1 \mathrm{n}}\right), \quad$ and $\left(\mathrm{w}_{31}, \mathrm{w}_{32}, \ldots \mathrm{w}_{3 \mathrm{n}}\right)$ respectively. The hidden layer to output layers weights are represented as $\left(\mathrm{w}_{211}, \mathrm{w}_{221}, \ldots . \mathrm{w}_{2 \mathrm{n} 1}\right)$. The output of the node is specified as (Y).

\section{Process for Training algorithm}

Process 1: Here, the weight of each neuron is assigned randomly for learning the network. The minimum and maximum weight (i.e.,) $\mathrm{W}=\left(\mathrm{w}_{\min }, \mathrm{W}_{\max }\right)$ of the interval range is specified as $(0,1)$.

Process 2: In the section, the BP error is determined. Here, the BP error is evaluated using the GSA. In the GSA, the optimized parameter of ANN is achieved while minimizing the BP error function.

\section{A) Back propagation error minimization using GSA technique}

Based on the law of gravity and mass interactions, GSA is a recently developed stochastic search algorithm. In GSA, the search agents are a collection of masses which interact with each other based on the Newtonian gravity and the laws of motion, totally different from other well-known population-based optimization method inspired by swarm behaviours in nature $[21,22,23]$. In the section, a GSA is used for improving the performance of ANN and reducing the BP error of the network. From the network study, the inputs, weights, and corresponding outputs of the system are found out. Based on the network output, the BP error is evaluated. The inputs of the GSA are the current and their corresponding network output, weights respectively. Here, the inputs are considered as the agents. The minimized BP error can be evaluated from the inputs. Based on evaluated inputs, the optimal outputs are determined. The procedure of the proposed algorithm is concisely explained as follows:

\section{Procedure of proposed algorithm}

1. In the section, inputs are the current and their corresponding network output, weights initiated randomly. Here, the inputs are considered as the agents. The position of agents are defined by the following equation,

$$
S=\left(s_{i}^{1}, \ldots, s_{i}^{d}, \ldots s_{i}^{n}\right)
$$

Where, $n$ is the search space dimension of the problem, $s_{i}^{d}$ is the position of the $\mathrm{i}^{\text {th }}$ agent in the $\mathrm{d}^{\text {th }}$ dimension.

2. The fitness function of agents is evaluated as their minimum range of $\mathrm{BP}$ error. The minimized error function of the network is given to the network. The fitness function of the agent is calculated as follows:

$$
F_{i}=\min \left(B P_{\text {error }}\right)
$$

$$
\text { Where, } B P_{\text {error }}=\sum_{i=1}^{n}\left(Y_{\text {targ } e t}^{i}-Y_{\text {output }}^{i}\right)^{2}
$$

From the above equation, the $\mathrm{Y}_{\text {target }}$ is the network target of the node and $\mathrm{Y}_{\text {out }}$ is the current output of the network.

After that, force of the agent is calculated.

3. The mass of agents are defined randomly and determine the forces of each agent. Here, the force acting on mass I from mass $j$ can be determined by,

$$
f_{i j}^{d}(k)=g(t)\left(\frac{M_{i}(k) * M_{j}(k)}{r_{i j}(k)+\epsilon}\right)\left(s_{j}^{d}(k)-s_{i}^{d}(k)\right)
$$

Where, $\mathrm{M}_{\mathrm{i}}(\mathrm{k})$ and $\mathrm{M}_{\mathrm{j}}(\mathrm{k})$ are masses of the agent $\mathrm{i}$ and $\mathrm{j}$. Here, $\mathrm{g}(\mathrm{k})$ is the gravitational constant, $\in$ is the small constant and $\mathrm{r}_{\mathrm{ij}}(\mathrm{k})$ is the Euclidian distance between $\mathrm{i}^{\text {th }}$ and $j^{\text {th }}$ agents. Calculate the gravitational constant of the agent using the following formula,

$$
g(k)=g_{0} * e^{\left(\frac{-\alpha k}{t_{r}}\right)}
$$

From the above equation, $t_{r}$ is the total iterations of the algorithm, $g_{0}$ is the initial value and $\alpha$ is the user specified constant.

4. The total force acts on the agent in $\mathrm{k}^{\text {th }}$ dimension is calculated as follows,

$$
f_{i}^{d}(k t)=\sum_{i=1 j \neq i}^{N} \operatorname{rand}_{i} f_{i j}^{d}(k)
$$

Where, $\operatorname{rand}_{\mathrm{j}}$ is a random number in the interval $[0,1]$ and the acceleration is calculated. 
5. Acceleration of any mass is equal to the force acted on the system divided by mass of inertia

$$
\alpha_{i}^{d}(k)=\frac{f_{i}^{d}(k)}{M_{i}(k)}
$$

6. New positions of the agents and gravitational constant $\&$ inertia masses are updated by the following equations.

$$
\begin{gathered}
m_{i}(k)=\frac{f i t_{i}(k)-\operatorname{worst}(k)}{\operatorname{best}(k)-\operatorname{worst}(k)} \\
M_{i}(k)=\frac{m_{i}(k)}{\sum_{j=1}^{N} m_{j}(k)}
\end{gathered}
$$

Where, fit $(\mathrm{k})$ represents the fitness value of the $\mathrm{i}^{\text {th }}$ agent at iteration $\mathrm{k}$.

7. Velocity of each mass is calculated, the new position of the masses could be considered Updating the agent's velocity and position using the following equation,

$$
V_{i}^{d}(k+1)=\operatorname{rand} \times v_{i}^{d}(k)+\alpha_{i}^{d}(k)
$$

When acceleration and velocity of each mass are calculated then new positions of the masses could be considered as follows

$$
s_{i}^{d}(k+1)=s_{i}^{d}(k)+v_{i}^{d}(k+1)
$$

Where, $V_{i}^{d}(k)$ and $s_{i}^{d}(k)$ are the velocity and position of an agent at the $\mathrm{k}$ time and dimension, random $_{i}$ is the random number at the interval at $[0$, $1]$.

8. The maximum iteration is reached, then the process is terminated otherwise repeat the step3-8. Here, the minimized BP error values are calculated and their corresponding inputs are noted. Based on the fitness function, the ANN is optimally trained and gets the optimal outputs and the corresponding back propagation error of the network is calculated.

Process 3: From the neural network, the current output is determined by following them,

$$
Y_{\text {out }}=\beta+\sum_{n=1}^{N} w_{2 n} Y_{i}(n)
$$

Here, $w_{i j}$ is the weight of the $i-j$ link of the network. Then, $y_{i}$ is the output of $i^{\text {th }}$ hidden neuron. Also, find out the change in weights based on the obtained BP error.

Process 4: Determine the bias (or) activation function of the network.

$$
Y_{i}(n)=\frac{1}{1+\exp \left(-w_{1 n} * Y_{i}(n)\right)}
$$

Process 5: The new weights of the each neurons of the network are updated by using the following equation,

$$
w_{n e w}=w_{\text {prev }}+\Delta w
$$

Here, $w_{n e w}$ is the new weight, $w_{\text {prev }}$ is the previous weight and $\Delta w$ is the change of weight of each output.

Process 6: Using the following equation, change of weight in the network is evaluated.

$$
\Delta w=\delta . Y_{\text {out }} \text {. BP } \text { error }
$$

In Eq. (22), $\delta$ is the learning rate. Repeat the above steps till the $\mathrm{BP}_{\text {error }}$ gets minimized $\mathrm{BP}_{\text {error }}<0.1$. Once the neural network training process is completed, the network is trained well for classifying the internal fault of the synchronous generator [24]. The detailed analysis of the proposed method is described in the following section.

\section{Results and Discussion}

The proposed technique is implemented in MATLAB /Simulink platform and their performances are evaluated.

The performances of the proposed method is evaluated and compared with the DWT-ANN with GA and DWT-ANN techniques and also the statistical measures such as accuracy, sensitivity and specificity values are analyzed.

Table 1. Implementation parameters

\begin{tabular}{|c|c|}
\hline Parameter & Rated values \\
\hline Rated power & 200MVA \\
\hline Rated voltage & $13.8 \mathrm{KV}$ \\
\hline Rated frequency & $50 \mathrm{~Hz}$ \\
\hline $\begin{array}{c}\text { Stator resistance } \\
\text { d-axis synchronous } \\
\text { reactance }\end{array}$ & 2.854 \\
\hline $\begin{array}{c}\text { q-axis synchronous } \\
\text { reactance }\end{array}$ & 0.474 \\
\hline $\begin{array}{c}\text { d-axis transient } \\
\text { reactance }\end{array}$ & 0.252 \\
\hline $\begin{array}{c}\text { d-axis transient } \\
\text { reactance }\end{array}$ & 0.243 \\
\hline
\end{tabular}

Initially, in the normal conditions, the synchronous generator, the stator winding current is analyzed. Later, the fault is created in the 
synchronous generator stator windings [23, 24]. Here, the internal fault is analyzed. In the internal faults, the performance of $\mathrm{AB}, \mathrm{BC}, \mathrm{CA}, \mathrm{ABC}$ faults are analyzed.

\section{Analysis of Internal fault}

The internal faults like $\mathrm{AB}, \mathrm{BC}, \mathrm{CA}$ and $\mathrm{ABC}$ faults synchronous generator are considered here. The synchronous generators are subjected to the most common type of fault which is the stator ground fault. Stator ground faults results due to insulation degradation in the winding along with environmental influence such as moisture or oil contamination with dirt which settles on the overhang portion of the winding. The DWT technique is applied to the current signals obtained at the terminals of synchronous generator. From the output of the DWT, the features are extracted. After that, these trained using the enhanced ANN technique. In figure 3, the illustrations of the internal faults are detailed.

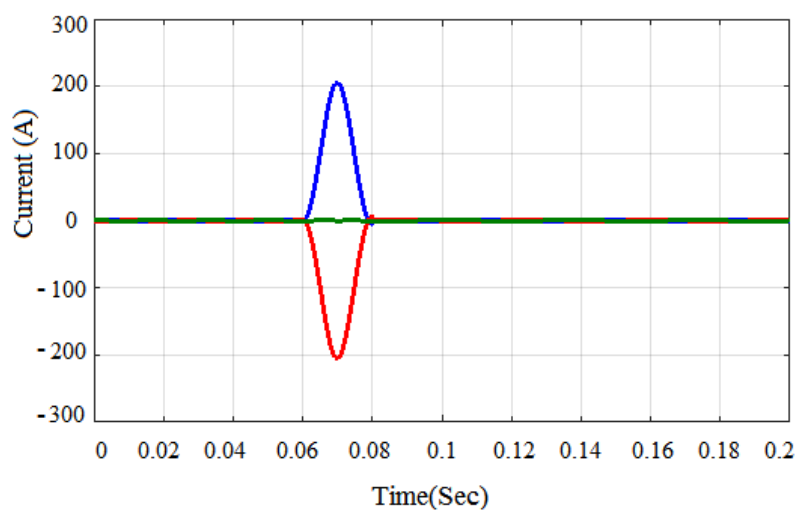

(a)

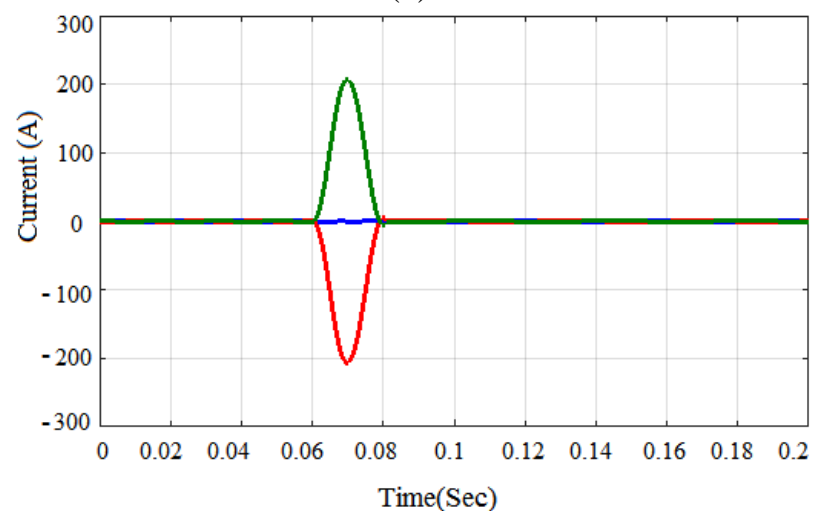

(b)

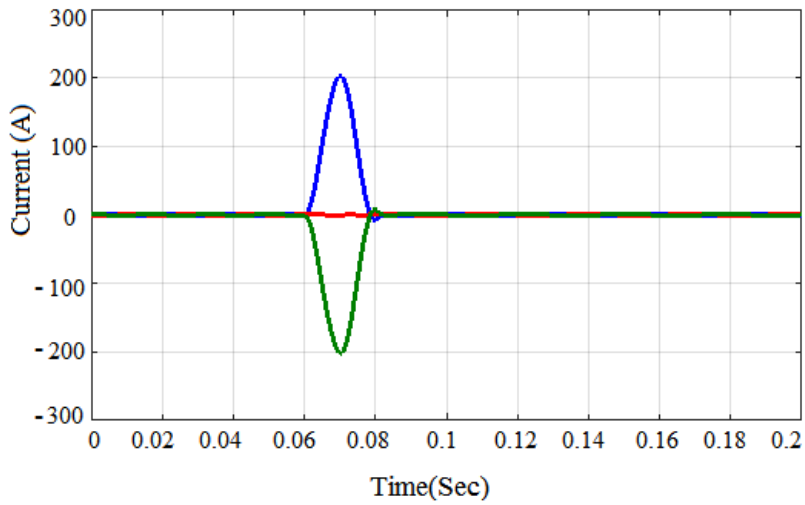

(c)

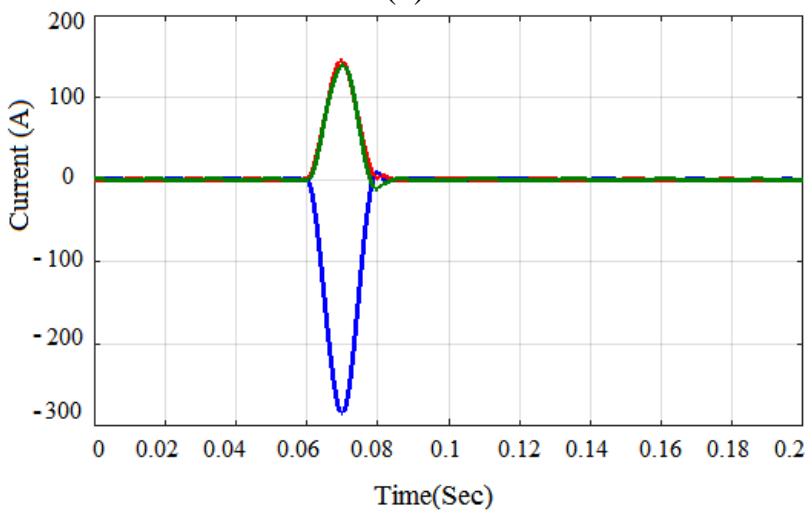

(d)

Figure 3: Analysis of synchronous generator internal faults in (a) $\mathrm{AB}$ (b) $\mathrm{BC}$ (c) $\mathrm{CA}$ and (d) $\mathrm{ABC}$
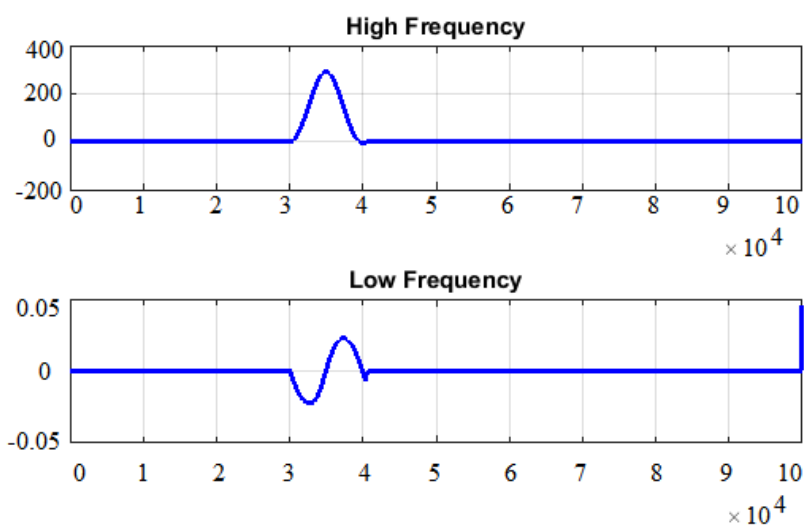

(a)
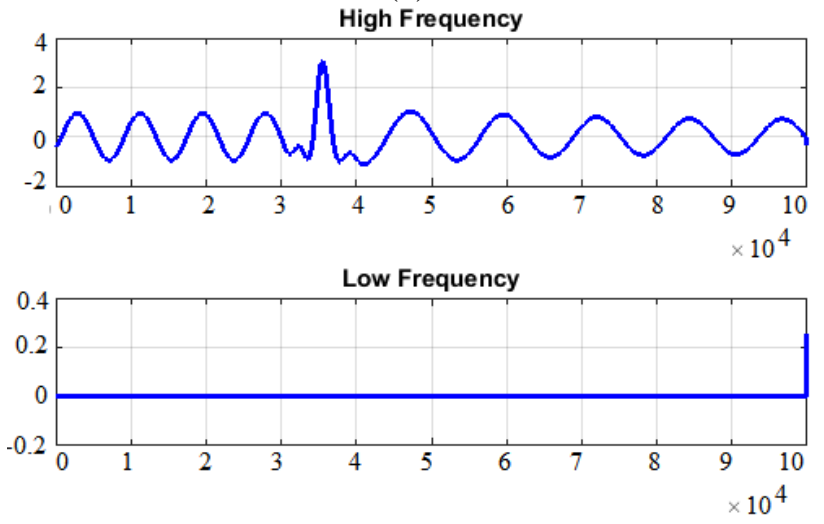

(b) 

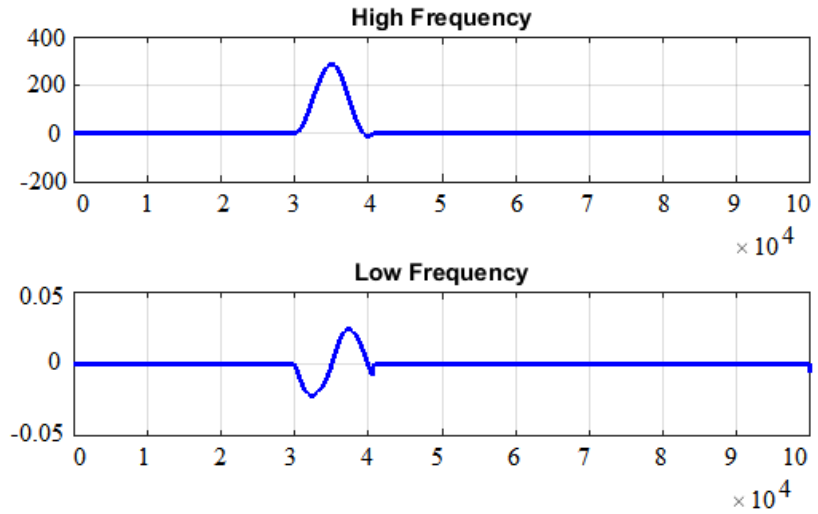

(c)
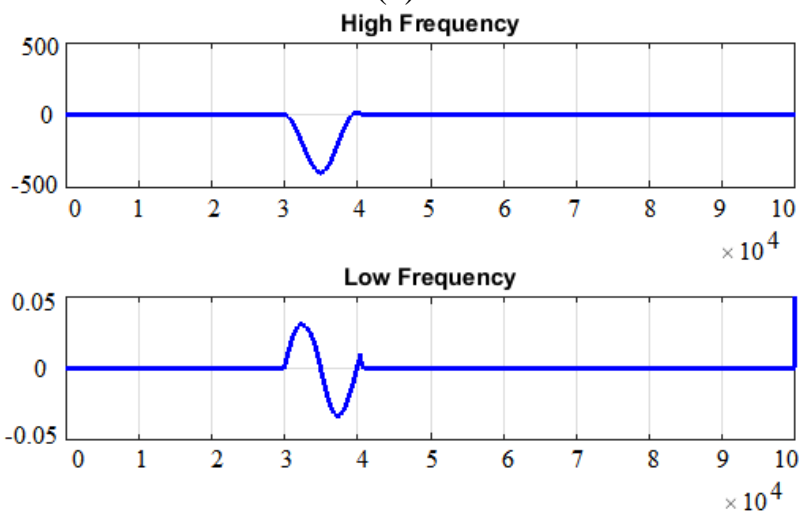

(d)

Figure.4 DWT analysis of internal faults in (a) AB (b) BC (c) $\mathrm{CA}$ and (d) $\mathrm{ABC}$

Then the low and high frequency signals of DWT are applied to the input of the enhanced ANN network. The enhanced ANNN is trained with the low and high frequency data and the faults are identified. The analysis of case 2 are specified in the below section.

\section{Analysis of Statistical measures}

The statistical measures such as accuracy, sensitivity and specificity are analyzed, in this section. The fault types are correctly classified, by using the proposed controller [25].

Table 2. Description of TP, TN, FP and FN

\begin{tabular}{|c|c|c|c|}
\hline \multicolumn{2}{|c|}{$\begin{array}{c}\text { Descriptions of } \\
\text { signals }\end{array}$} & \multicolumn{2}{c|}{ Testing results } \\
\cline { 2 - 4 } & $\begin{array}{c}\text { Faulty } \\
\text { signal }\end{array}$ & $\begin{array}{c}\text { Normal } \\
\text { signal }\end{array}$ \\
\hline \multirow{2}{*}{$\begin{array}{c}\text { Input } \\
\text { Conditions }\end{array}$} & $\begin{array}{c}\text { Faulty } \\
\text { signal }\end{array}$ & TP & FN \\
\cline { 2 - 4 } & $\begin{array}{c}\text { Normal } \\
\text { signal }\end{array}$ & FP & TN \\
\hline
\end{tabular}

Table 3. Accuracy, Sensitivity and Specificity for Proposed method

\begin{tabular}{|c|c|c|c|c|c|c|c|}
\hline \multirow{2}{*}{$\begin{array}{l}\text { Signal } \\
\text { analysis }\end{array}$} & \multicolumn{7}{|c|}{$\begin{array}{l}\text { Internal faults analysis using } \\
\text { proposed method }\end{array}$} \\
\hline & $\mathrm{TP}$ & $\mathrm{FP}$ & $\mathrm{FN}$ & $\mathrm{TN}$ & $\mathrm{A}$ & $\mathrm{Se}$ & $\mathrm{Sp}$ \\
\hline $\mathrm{AB}$ & 8 & 1 & 2 & 9 & 0.85 & 0.8 & 0.9 \\
\hline $\mathrm{BC}$ & 9 & 1 & 1 & 9 & 0.9 & 0.9 & 0.9 \\
\hline $\mathrm{CA}$ & 9 & 2 & 1 & 8 & 0.85 & 0.9 & 0.8 \\
\hline $\mathrm{ABC}$ & 10 & 2 & 0 & 8 & 0.9 & 1 & 0.8 \\
\hline Normal & 9 & 2 & 1 & 8 & 0.85 & 0.9 & 0.8 \\
\hline
\end{tabular}

Then, the statistical performance of the proposed method is compared with the existing methods like DWT-ANN-GA and DWT-ANN respectively. The Accuracy (A), Sensitivity (Se) and Specificity (Sp) of the proposed technique is analyzed from the True positive (TP), False positive (FP), True negative $(\mathrm{TN})$ and False negative (FN) values.

From the above table 1, the TP, FP, TN and FN are described as the $\mathrm{AB}$ fault is correctly identified as a faulty signal, Normal signal is incorrectly identified as fault signal, Normal signal is correctly identified as normal and $\mathrm{AB}$ fault signal incorrectly identified as normal respectively. Likewise, the other types of faults are described and the accuracy, sensitivity \& specificity values are calculated by using the following formula,

\section{Accuracy:}

$$
A=\frac{T P+T N}{T P+F P+T N+F N}
$$

Sensitivity:

$$
S e=\frac{T P}{(T P+F N)}
$$

Specificity:

$$
S p=\frac{T N}{(F P+T N)}
$$

The fault is detected and classified their types, from the output of proposed method. Then, from the testing output of enhanced ANN method, the TP, $\mathrm{TN}, \mathrm{FP}$ and FN are assessed. Then the performance of proposed technique (DWT-ANN with GSA) is determined and compared with DWT-ANN, GA and DWT-ANN techniques. In Table 2, 3, 4, the evaluated output of proposed technique is tabulated. 


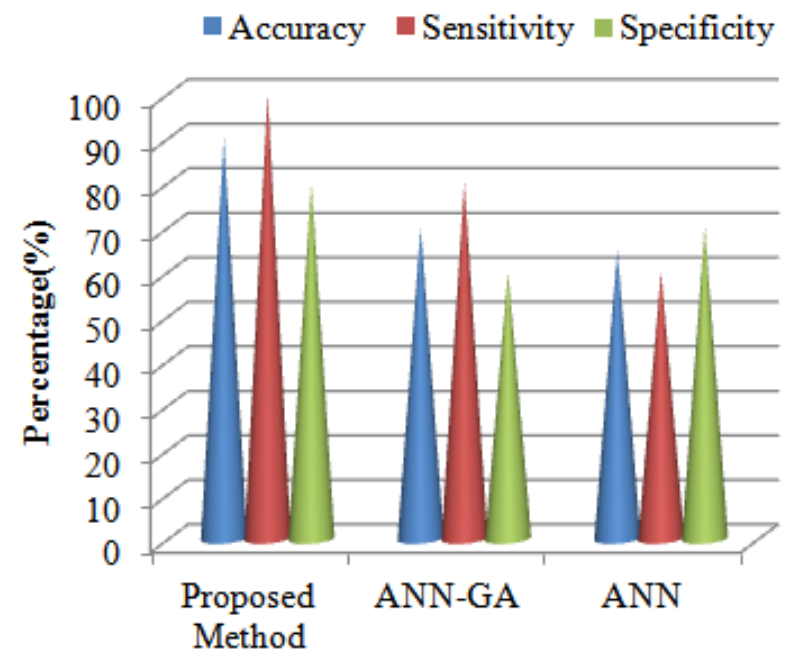

Figure.5 Comparison analysis of statistical measures in internal fault

Table 4. Accuracy, Sensitivity and Specificity for DWTANN with GA method

\begin{tabular}{|c|c|c|c|c|c|c|c|}
\hline \multirow{2}{*}{$\begin{array}{l}\text { Signal } \\
\text { analysis }\end{array}$} & \multicolumn{7}{|c|}{$\begin{array}{l}\text { Internal faults analysis using } \\
\text { DWT-ANN with GA }\end{array}$} \\
\hline & $\mathrm{TP}$ & FP & $\mathrm{FN}$ & $\mathrm{TN}$ & A & $\mathrm{Se}$ & $\mathrm{Sp}$ \\
\hline $\mathrm{AB}$ & 7 & 2 & 3 & 8 & 0.75 & 0.7 & 0.8 \\
\hline $\mathrm{BC}$ & 7 & 3 & 3 & 7 & 0.7 & 0.7 & 0.7 \\
\hline $\mathrm{CA}$ & 6 & 4 & 4 & 6 & 0.6 & 0.6 & 0.6 \\
\hline $\mathrm{ABC}$ & 8 & 4 & 2 & 6 & 0.7 & 0.8 & 0.6 \\
\hline Normal & 9 & 2 & 1 & 8 & 0.85 & 0.9 & 0.8 \\
\hline
\end{tabular}

In Figure 5, it is shown that for the proposed technique and other techniques namely DWT-ANN with GA and DWT-ANN, the comparison of the performance measures such as accuracy, sensitivity and specificity. However, seeing the accuracy value of the three techniques, the proposed technique generated higher accuracy than the other techniques and it is about $15-23 \%$. Likewise, the sensitivity and specificity values are assessed. The specificity value is also higher for the proposed technique which in turn shows the effectiveness of the proposed technique while categorizing the internal faults.
From the comparative analysis, the Accuracy (A), Sensitivity (Se) and Specificity (Sp) are analyzed at different inputs. The analysis results are showed the proposed technique (DWT-ANN with GSA) is better than that compared to DWT-ANN with GA and DWT-ANN.

\section{Conclusion}

In this paper, we have studied DWT and ANN with GSA technique, fault detection and classification in the synchronous generator. In this, for extracting the features of the input signals such as low and high frequency components, the internal faulty signals are examined and DWT is offered. And for the classifying of the signals to check whether it is faulty or not the Enhanced ANN is used. The proposed technique for identifying the types of faults was experimented with MATLAB/Simulink platform. The effectiveness of the proposed method was analyzed and evaluated. By means of statistical measures such as accuracy, sensitivity \& specificity, the performance of the suggested work was authenticated and additionally it was compared with DWT-ANN with GA and DWTANN methods. It is demonstrated that the suggested method was better using comparison performances and ahead of other methods in terms of statistical measures. In future, the performances of synchronous generator will be analyzed by utilizing the different types of faults. Moreover, the recent optimization technique will be utilized to detect and classify the faults of synchronous generator.

\section{References}

[1] C. A. Platero, F. Blazquez, P. Frias and D. Ramfrez, "Influence of Rotor Position in FRA Response for Detection of Insulation Failures in Salient-Pole Synchronous Machines", IEEE Transactions on Energy Conversion, Vol. 26, No. 2, pp. 671-676, 2011

[2] M. Amini, M. Davarpanah and M. S. Pasand, "A Novel Approach to Detect the Synchronous Generator Loss of Excitation", IEEE Transactions on Power Delivery, Vol. 30, No. 3, pp. 1429-1438, 2015

[3] N. M. A. Freire, J. O. Estima and A. J. M. Cardoso, "A New Approach for Current Sensor Fault Diagnosis in PMSG Drives for Wind Energy Conversion Systems", IEEE Transactions on Industry Applications, Vol. 50, No. 2, pp. 1206-1214, 2014

[4] F. R. Blanquez, M. Aranda, E. Rebollo, F. Blazquez, and C. A. Platero, "New Fault-Resistance Estimation Algorithm for Rotor-Winding Ground-Fault On-Line Location in Synchronous Machines with Static Excitation", IEEE Transactions on Industrial Electronics, Vol. 62, No. 3, pp. 1901-1911, 2015 
[5] F. R. Blanquez, C. A. Platero, E. Rebollo and F. Blazquez, "On-line stator ground-fault location method for synchronous generators based on $100 \%$ stator low-frequency injection protection", Electric Power Systems Research, Vol. 125, pp. 34-44, 2015

[6] M. V. D. Geest, H. Polinder, J. A. Ferreira, A. Veltman, J. J. Wolmarans and N. Tsiara, "Analysis and Neutral Voltage Based Detection of Inter-Turn Faults in High-Speed Permanent Magnet Machines with Parallel Strands", IEEE Transactions on Industrial Electronics, Vol. 62, No. 6, pp. 3862-3873, 2015

[7] K. Liu and Z. Q. Zhu, "Position-Offset-Based Parameter Estimation Using the Adaline NN for Condition Monitoring of Permanent-Magnet Synchronous Machines", IEEE Transactions on Industrial Electronics, Vol. 62, No. 4, pp. 2372-2383, 2015

[8] B. Aubert, J. Regnier, S. Caux and D. Alejo, "Kalman-Filter-Based Indicator for Online Interturn Short Circuits Detection in Permanent-Magnet Synchronous Generators", IEEE Transactions on Industrial Electronics, Vol. 62, No. 3, pp. 1921-1930, 2015

[9] I. Jlassi, J. O. Estima, S. K. E. Khil, N. M. Bellaaj and A. J. M. Cardoso, "Multiple Open-Circuit Faults Diagnosis in Back-to-Back Converters of PMSG Drives for Wind Turbine Systems", IEEE Transactions on Power Electronics, Vol. 30, No. 5, pp. 2689-2702, 2015

[10]H. Yaghobi, "Impact of static synchronous compensator on flux-based synchronous generator loss of excitation protection", IET Generation on Transmission \& Distribution, Vol. 9, No. 9, pp. 874883,2015

[11]S. Hemmati, S. S. Kojoori, S. Saied and T. A. Lipo, "Modelling and experimental validation of internal short-circuit fault in salient-pole synchronous machines using numerical gap function including stator and rotor core saturation", IET Electric Power Applications, Vol. 7, No. 5, pp. 391-399, 2013

[12]R. Gopinath, C. S. Kumar, K. I. Ramachandran, V. Upendranath and P. V. R. Sai Kiran, "Intelligent Fault Diagnosis of Synchronous Generators", Expert Systems with Applications, Vol. 45, pp. 142-149, 2016

[13]X. L. Zhang, W. Chen, B. J. Wang and X. F. Chen, "Intelligent fault diagnosis of rotating machinery using support vector machine with ant colony algorithm for synchronous feature selection and parameter optimization", Neuro-computing, Vol. 167, pp. 260-279, 2015

[14]P. M. Vahdati, H. Seyedi, A. Arjmandi and S. Moradi, "Synchronous generator stator protection using a fuzzy logic-based voltage-controlled over current protection scheme", International Transactions on Electrical Energy Systems, Vol. 25, pp. 326-347, 2015

[15]G. Niu, Y. Zhao, M. Defoort and M. Pecht, "Fault diagnosis of locomotive electro-pneumatic brake through uncertain bond graph modeling and robust online monitoring", Mechanical Systems and Signal Processing, Vol. 50, pp. 676-691, 2015

[16]A. Ali, A. Q. Khan, B. Hussain, M. T. Raza and M. Arif, "Fault modelling and detection in power generation, transmission and distribution systems", IET Generation, Transmission \& Distribution, pp. 110,2015

[17]A. Safari, C. V. Niras and Y. Kong, "Powerperformance enhancement of two-dimensional RNSbased DWT image processor using static voltage scaling", VLSI Journal of Integration, Vol. 53, pp. 145-156, March 2016

[18]B.Vidyasagar, SS.Tulasiram, "Diagnosis of Incipient Faults Using Wavelet Packet Decomposition in Synchronous Generators", International Journal of Industrial Electronics and Electrical Engineering, Vol. 4, No. 4, pp. 28-35

[19]H. Fathabadi, "Power distribution network reconfiguration for power loss minimization using novel dynamic fuzzy c-means (dFCM) clustering based ANN approach", International Journal of Electrical Power \& Energy Systems, Vol. 78, pp. 96107, June 2016

[20]T. Xu, B. Venkatesh, C. Opathella and B. N. Singh, "Artificial neural network model of photovoltaic generator for power flow analysis in PSS®SINCAL", IET Generation, Transmission \& Distribution, Vol. 8, No. 7, pp. 1346-1353, 2014

[21]T. Niknam, M. R. Narimani, R. A. Abarghooee and B. B. Firouzi, "Multiobjective Optimal Reactive Power Dispatch and Voltage Control: A New OppositionBased Self-Adaptive Modified Gravitational Search Algorithm", IEEE Systems Journal, Vol. 7, No. 4, pp. 742-753, 2013

[22]S. D. Beigvand, H. Abdi and M. L. Scala, "Combined heat and power economic dispatch problem using gravitational search algorithm", Electric Power Systems Research, Vol. 133, pp. 160-172, April 2016

[23]S. M. A. Elazim and E. S. Ali, "Optimal SSSC design for damping power systems oscillations via Gravitational Search Algorithm", International Journal of Electrical Power \& Energy Systems, Vol. 82, pp. 161-168, November 2016

[24]B. Vidyasagar and S. S. Tulasiram, "Fault diagnosis of Synchronous Generator using Neural Network Techniqes", International Journal of Ethics in Engineering \& Management Education, ISSN: 23484748, Vol. 1, No. 2, February 2014

[25]N. Yadaiah and N. Ravi, "Statistical method for fault detection in synchronous generators", In proceedings of International Conference on Computer Communication and Informatics (ICCCI), pp. 1-4, 2012 